\title{
Mob/oriT, a mobilizable site-specific recombination system for unmarked genetic manipulation in Bacillus thuringiensis and Bacillus cereus
}

Pengxia Wang ${ }^{\dagger}$, Yiguang Zhu ${ }^{\dagger}$, Yuyang Zhang, Chunyi Zhang, Jianyi Xu, Yun Deng, Donghai Peng, Lifang Ruan and Ming Sun*

\begin{abstract}
Background: Bacillus thuringiensis and Bacillus cereus are two important species in B. cereus group. The intensive study of these strains at the molecular level and construction of genetically modified bacteria requires the development of efficient genetic tools. To insert genes into or delete genes from bacterial chromosomes, marker-less manipulation methods were employed.

Results: We present a novel genetic manipulation method for $B$. thuringiensis and B. cereus strains that does not leave selection markers. Our approach takes advantage of the relaxase Mob02281 encoded by plasmid pBMB0228 from Bacillus thuringiensis. In addition to its mobilization function, this Mob protein can mediate recombination between oriT sites. The Mob02281 mobilization module was associated with a spectinomycin-resistance gene to form a MobSpc cassette, which was flanked by the core 24-bp oriT sequences from pBMB0228. A strain in which the wild-type chromosome was replaced with the modified copy containing the Mob-Spc cassette at the target locus was obtained via homologous recombination. Thus, the spectinomycin-resistance gene can be used to screen for Mob-Spc cassette integration mutants. Recombination between the two oriT sequences mediated by Mob02281, encoded by the MobSpc cassette, resulted in the excision of the Mob-Spc cassette, producing the desired chromosomal alteration without introducing unwanted selection markers. We used this system to generate an in-frame deletion of a $\operatorname{target}$ gene in $B$. thuringiensis as well as a gene located in an operon of $B$. cereus. Moreover, we demonstrated that this system can be used to introduce a single gene or an expression cassette of interest in B. thuringiensis.
\end{abstract}

Conclusion: The Mob/oriT recombination system provides an efficient method for unmarked genetic manipulation and for constructing genetically modified bacteria of $B$. thuringiensis and B. cereus. Our method extends the available genetic tools for $B$. thuringiensis and B. cereus strains.

Keywords: Bacillus thuringiensis, Bacillus cereus, Relaxase, Mob protein, Site-specific recombination, Conjugation

\section{Background}

The Bacillus cereus group currently includes eight closely related species: $B$. anthracis, B. cereus, B. thuringiensis, B. cytotoxicus, B. mycoides, B. pseudomycoides, B.

\footnotetext{
*Correspondence: m98sun@mail.hzau.edu.cn

${ }^{\dagger}$ Pengxia Wang and Yiguang Zhu contributed equally to this work State Key Laboratory of Agricultural Microbiology, College of Life Science and Technology, Huazhong Agricultural University, Wuhan, People's Republic of China
}

weihenstephanensis and B. toyenensis [1-3]. These microbial organisms are widespread in natural environments and have high economic, medical, and biodefense importance. $B$. cereus is a well-known food poisoning bacterium; B. thuringiensis, which produces insecticidal crystal proteins and is widely used to control agricultural pests [1]. The development of global systems biology tools, such as genome sequencing technologies and DNA microarrays, has stimulated the study of functional genes, 
biosynthetic gene clusters, and other aspects of cell biology $[4,5]$. The intensive study of $B$. thuringiensis and $B$. cereus strains at the molecular level and construction of genetically modified bacteria requires the development of efficient genetic tools, especially vectors for targeted gene deletions and insertions.

Gene inactivation via the insertion of an antibioticresistance marker into a target gene is frequently used for functional analyses. However, when a single strain is manipulated repeatedly, the small number of convenient selectable markers limits the application of this approach. Moreover, since operons are common in bacterial genomes, the insertion of a marker gene can alter the expression of adjacent genes. Third, genetically modified bacteria containing antibiotic-resistance genes are not desirable in production systems, because the antibiotic resistance represents a contaminant of the final product and carries the risk of horizontal gene transfer of antibiotic-resistance genes to the environment. Therefore, a method of inserting genes into or deleting genes from bacterial chromosomes without leaving selection markers is a significant advantage. The approaches have been established in some microorganisms by the utilization of recombinase-mediated recombination systems. Various site-specific recombination systems are used, e.g., the FLP/FRT recombination system of Saccharomyces cerevisiae [6-8], the Cre/loxP recombination system from Escherichia coli bacteriophage P1 $[9,10]$, and the Xer/dif recombination system based on endogenous Xer recombinases $[11,12]$. In all of these systems, two different or identical recognition sites act as the DNA substrate for the recombinase, and a single recombination site remains within the target locus. The FLP/FRT and Cre/loxP systems require many time-consuming steps, including the construction of an exogenous recombinase FLP or Cre in a replicative plasmid, the introduction of the plasmid, recombinase expression in the transformant, and elimination of the replicative plasmid $[7,10]$. Moreover, the $\mathrm{FLP} / F R T$ system is not efficient in some slow-growing bacteria [8]. The Xer/dif system utilizes the endogenous recombinase Xer to recognize and resolve dif sites to excise antibiotic resistance genes. However, Xer recombinases and the relevant dif sites differ among bacteria, and some Xer systems include two recombinases [13], while others include one recombinase $[12,14]$.

Relaxase and oriT are critical for the transfer of both conjugative plasmids and mobilizable plasmids [15]. The origin of transfer, or oriT sequence, is the only component that should be provided by the mobilizable plasmid in cis. The relaxase, which is also called a Mob protein in a mobilizable plasmid, catalyzes the initial and final stages of conjugation. In addition, to their nicking activity necessary for mobilizing plasmids, certain relaxases can catalyze recombination between two oriT sequences, including NikB (R64), TrwC (R388), MobA (R1162), Mob02281 (pBMB0228), and Mob02282 (pBMB0228) [16-19]. This recombination event can mediate the deletion of a target DNA region between two oriT sequences. Accordingly, owing to their functions in conjugation and recombination, relaxases have been used for various purposes, e.g., to construct transfer vectors $[20,21]$ and for oriT-directed cloning of large bacterial genomic regions [22]. However, relaxase-mediated mechanisms have not been used for unmarked genetic manipulation.

In a previous study, we confirmed that Mob02281 and Mob02282 not only have mobilization ability, but also mediate the resolution and formation of plasmid pBMB0228 from B. thuringiensis strain YBT-1518, the mechanism is oriT site-specific recombination [19]. Mob02281 has a higher mobilization efficiency and recombination ability than Mob02282 [19]; accordingly, we used it to develop novel molecular tools, and demonstrated the potential application of this method to other bacterial taxa.

\section{Results \\ Relaxase Mob02281 can transfer a target gene into $B$. thuringiensis and $B$. cereus strains}

To test whether Mob02281 can transfer a target gene into $B$. thuringiensis and B. cereus strains, the plasmid cry6AapHTMob02281, which contains cry6Aa as a reporter, was constructed. The cry $6 A a$ gene can produce rice-shaped crystals and encodes a $54-\mathrm{kDa}$ protein [23]. The plasmid pHTMob02281 was used in parallel and was treated similarly to cry6Aa-pHTMob02281. Using the conjugative plasmid pAW63::Tn5401, cry6Aa-pHTMob02281 and pHTMob02281 could be transferred to all recipient $B$. thuringiensis and $B$. cereus strains with a transfer efficiency ranging from $10^{-7}$ to $10^{-5}$ transconjugants/ donor (Table 1). Based on phase-contrast microscopy, all transconjugants containing cry6Aa-pHTMob02281 produced rice-shaped crystals, and SDS-PAGE showed that all transconjugants containing cry6Aa-pHTMob02281 produced parasporal crystals with a $54-\mathrm{kDa}$ band representing Cry6Aa (Fig. 1).

\section{Relaxase Mob02281 mediates the excision of the resistance marker between the 24-bp core oriT sites}

To decrease the influence of DNA remnants after Mob02281-mediated recombination, we identified the minimal oriT sequence required for Mob02281-mediated recombination. Using the previous recombination system [19], we performed a series of deletions in one or both oriTs (352-bp oriT1 and 370-bp oriT2) of the substrate plasmid pBMBT10. The pBMBmob1 plasmid containing Mob02281 was used as a helper plasmid. As 
Table 1 Transfer efficiency of cry6Aa-pHTMob02281 and pHTMob02281 in B. thuringiensis and B. cereus strains

\begin{tabular}{|c|c|c|c|c|c|}
\hline $\begin{array}{l}\text { Plasmid } \\
\text { donors }\end{array}$ & Recipients & Efficiency & Plasmid donors & Recipients & Efficiency \\
\hline \multirow{5}{*}{ cry6Aa-pHTMob02281 } & BMB171Str & $4.5 \times 10^{-5}$ & \multirow{5}{*}{ pHTMob02281 } & BMB171Str & $3.4 \times 10^{-5}$ \\
\hline & YBT1520Str & $8.2 \times 10^{-6}$ & & YBT1520Str & $9.3 \times 10^{-6}$ \\
\hline & CT-43::Kan & $3.0 \times 10^{-5}$ & & CT-43::Kan & $2.8 \times 10^{-5}$ \\
\hline & UW85R & $2.3 \times 10^{-5}$ & & UW85R & $2.7 \times 10^{-5}$ \\
\hline & BC10987Str & $5.3 \times 10^{-6}$ & & BC10987Str & $7.0 \times 10^{-6}$ \\
\hline
\end{tabular}

Transfer efficiency $=$ transconjugants/donor cells

shown in Fig. 2, deletions at the two oriT sites had different effects on recombination activity. Compared with the high recombination frequency observed when the substrate plasmid contains bp 1-352 of oriT1 and bp 1-370 of oriT2, the recombination frequency exhibited a sharp decrease when inverted repeat IR 1 and IR2 were deleted from oriT2. Surprisingly, it showed a relatively higher recombination frequency when the substrate plasmid contains bp 1-352 of oriT1 and bp 273-297 of oriT2 having only 24 bp spanning IR6. However, by remaining the 370 -bp oriT2 sequence unchanged, the deletion of oriT1 had a strong influence on recombination frequency.

When the substrate plasmid contains bp 258-282 of oriT1 and bp 273-297 of oriT2 (i.e., only 24 bp of inverted repeat sequences, including the nic site), recombination was observed (Fig. 3). A schematic diagram of substrate construction is shown in Fig. 3a. Additionally, fluorescent BMB171 (pBMBTmini + pBMBmob1) cells were observed under phase contrast microscopy and fluorescence microscopy after recombination (Fig. $3 \mathrm{~b}$ ). Single $\mathrm{Spc}^{\mathrm{S}}$ colonies were confirmed by a restriction analysis (Fig. 3c) and sequencing (Additional file 1: Fig. S1). When the cultures were grown for 20 generations, three $\mathrm{Spc}^{\mathrm{S}}$ colonies out of 200 total colonies were obtained. Although this was a low level of recombination, it was sufficient to obtain a recombined plasmid. With additional generations, more $\mathrm{Spc}^{\mathrm{S}}$ colonies were obtained. When the cultures were grown for 100 generations, nine $\mathrm{Spc}^{\mathrm{S}}$ colonies out of 100 colonies were obtained (Fig. 3d). These results indicate that the Mob02281/mini-oriT (24 bp of oriT1 and $24 \mathrm{bp}$ of oriT2) system can mediate target gene deletion.

\section{Construction of a Ts mobilizable site-specific} recombination vector for unmarked genetic manipulation We confirmed the mobilization ability of the Mob02281 module and site-specific recombination mediated by Mob02281. We then constructed a modular vector, pRecmob1-Ts, to facilitate the use of these components for the genetic manipulation of $B$. thuringiensis and B. cereus strains. The crucial element of this modular plasmid is the Mob-Spc cassette, which contains the Mob02281 mobilizable module and a spectinomycin-resistance gene, flanked by the two 24-bp oriT core sites in the same orientation and multiple restriction sites for subcloning the homologous arms of the target gene (Fig. 4).

\section{Construction of an integration vector for unmarked genetic manipulation}

To develop a universal integration vector for target gene or gene cluster expression in B. thuringiensis and $B$. cereus strains, a putative amylase gene was used because it is not essential and is conserved in $B$. thuringiensis and B. cereus. The amyE upstream and downstream homologous arms separated by the Mob-Spc cassette were constructed to disrupt amylase based on pRec-mob-Ts (Fig. 5a), generating the integration vector plasmid pBMB0260. Then, pBMB0260 was used to transform $B$. thuringiensis BMB171 by electroporation. BMB0260::MobSpc was obtained by using the spectinomycin-resistance gene as the selection marker. The unmarked mutant strain BMB0260 was then obtained by culturing BMB0260::MobSpc in LB medium at $28{ }^{\circ} \mathrm{C}$; after approximately 30 generations, two spectinomycin-sensitive colonies were obtained and confirmed by PCR using primers outside the homologous arms (Fig. 5b). The PCR product was sequenced to confirm that BMB0260 was constructed by replacing a 297-bp internal amyE gene fragment with a 36-bp segment that contains the 24-bp oriT and two 6-bp restriction sites (Fig. 5c).

We tested the $\alpha$-amylase activity of the amyE mutant strain BMB0260 using a starch-plate assay. Two single colonies of BMB0260 exhibited a circle indicating starch hydrolysis that was similar to that of BMB171, which carried an intact amylase gene (Additional file 1: Fig. S2). Based on the genome sequence analysis, we found that there are two putative amylase genes in most $B$. thuringiensis and $B$. cereus strains, such as BMB171_C1018 


\section{a}

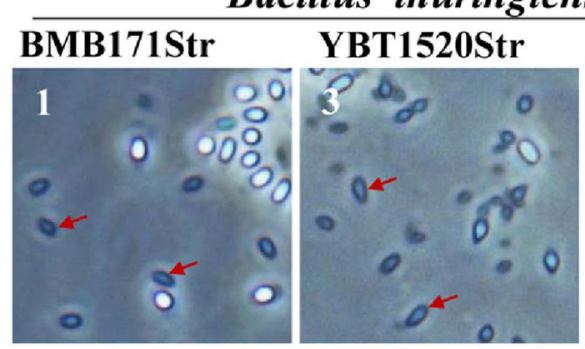

\section{Bacillus thuringiensis}

cry6Aa-pHTMob02281
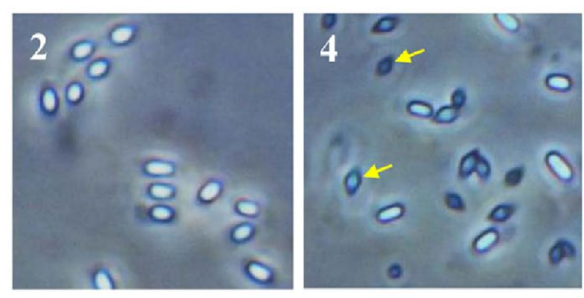

pHTMob02281

b

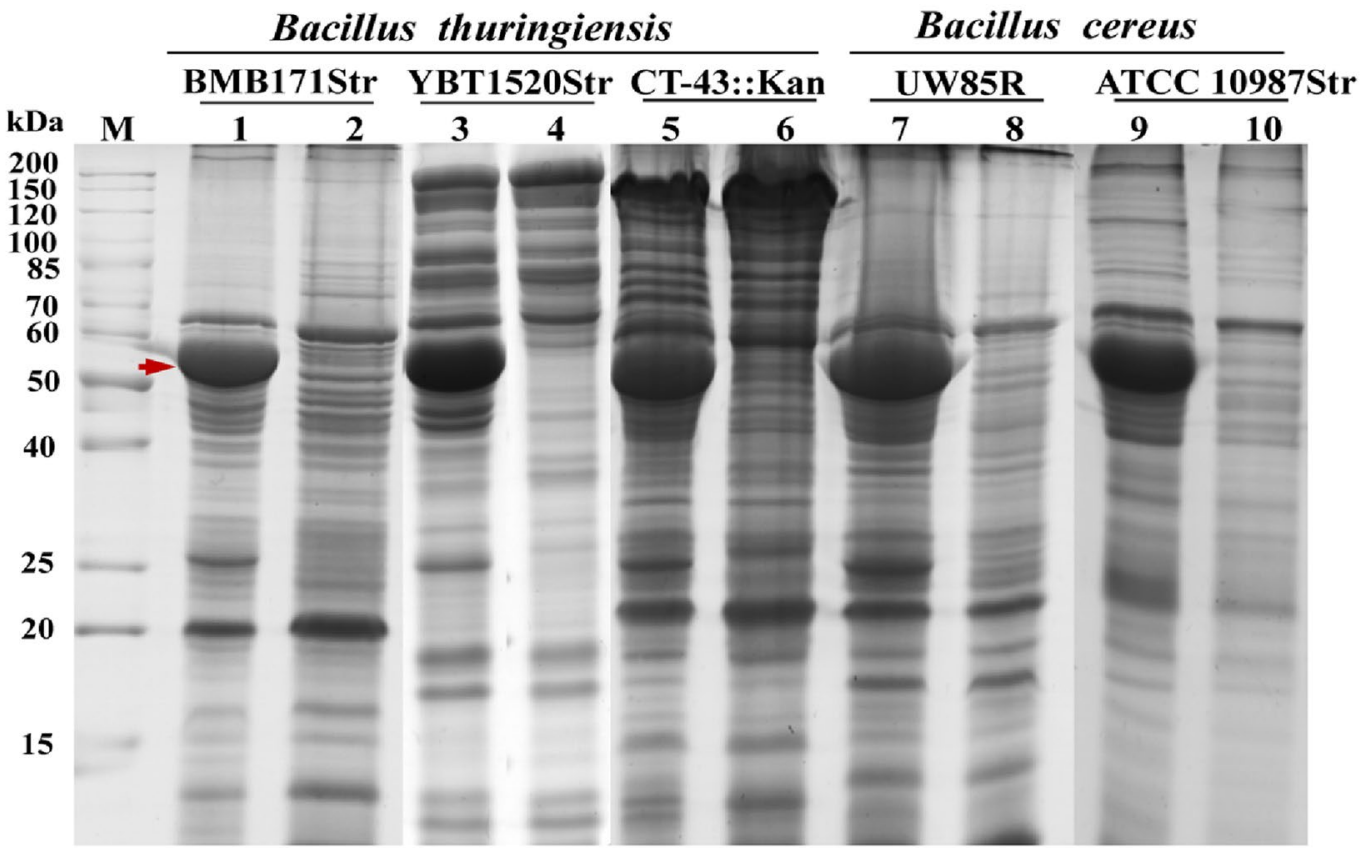

Fig. 1 Parasporal crystals and protein expression of cry6Aa in transconjugants. a Phase-contrast microscopy image of parasporal crystals and spores produced in transconjugants. The red arrow indicates the rice-shaped crystals encoded by cry6Aa. The yellow arrow indicates the diamond-shaped crystals encoded by the endogenous crystal genes for YBT1520Str [5] and CT43::Kan [40]. b Protein analysis of the transconjugants by SDS-PAGE. The red arrow indicates the 54-kDa protein produced by cry6Aa. 1 BMB171Str/cry6Aa-pHTMob02281. 2 BMB171Str/pHTMob02281. 3 YBT1520Str/cry6AapHTMob02281. 4 YBT-1520Str/pHTMob02281. 5 CT-43::Kan/cry6Aa-pHTMob02281. 6 CT-43::Kan/pHTMob02281. 7 UW85R/cry6Aa-pHTMob02281. 8 UW85R/pHTMob02281. 9 ATCC 10987Str/cry6Aa-pHTMob02281. 10 ATCC 10987Str/pHTMob02281. M Protein molecular weight marker

encoding alpha-amylase and BMB171_C3160 encoding cytoplasmic alpha-amylase in BMB171. The deleted BMB171_C1018 gene was a putative alpha amylase, but its $\alpha$-amylase activity has not been confirmed. This result suggests that this amylase gene has very low or no activity.
Unmarked integration of cry5Ba or cry2Aa at the amylase locus

The plasmids pBMB0261 and pBMB0262 respectively containing cry $5 \mathrm{Ba}$ gene and $\operatorname{cry} 2 \mathrm{Aa}$ expression cassette were used to transform B. thuringiensis BMB171 (Fig. 6a, b). After the Mob-spc insertion mutants were obtained, 


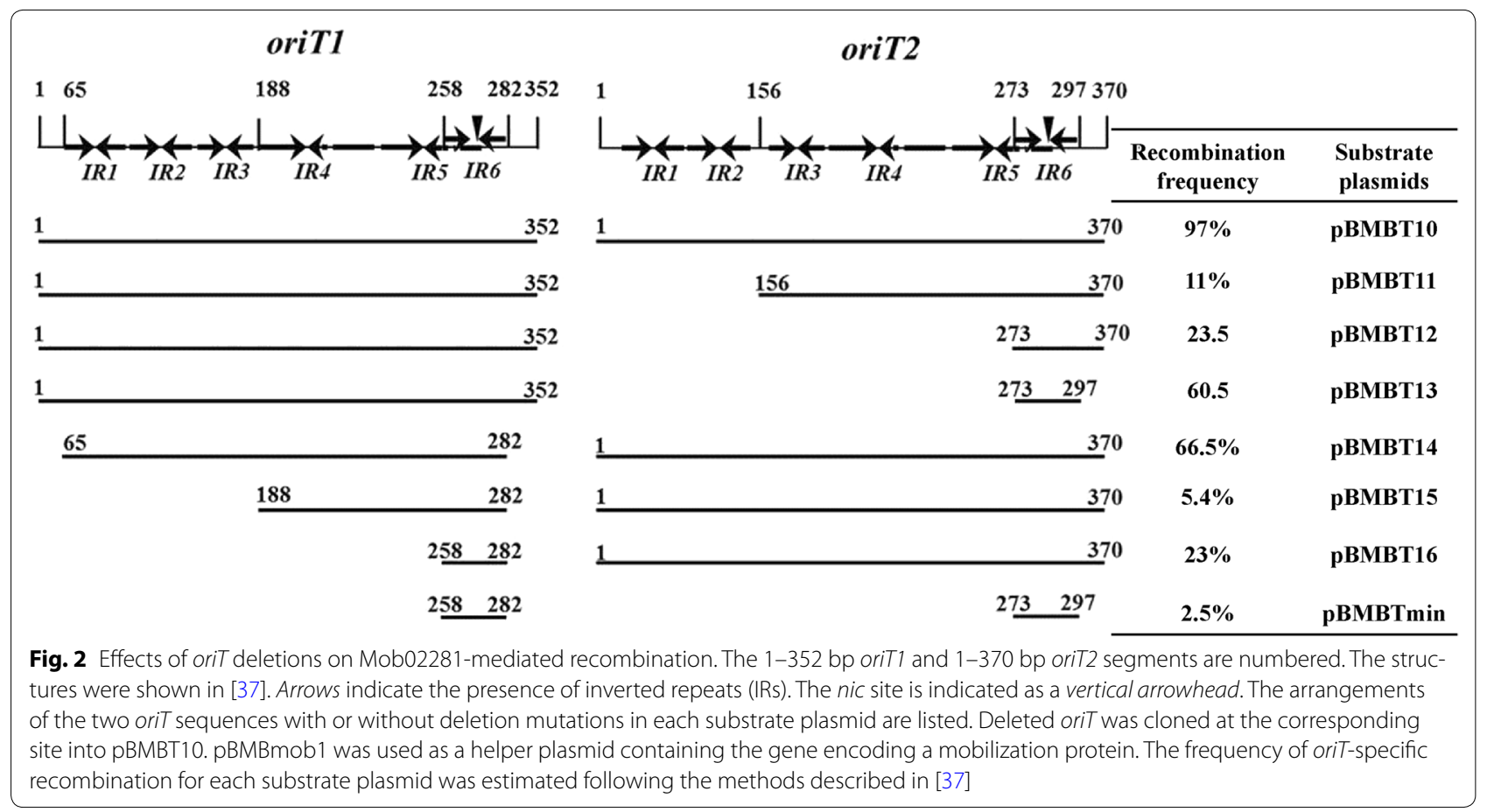

the unmarked integration mutants were generated following similar methods to those for amyE disruption, and a similar Mob-spc cassette excision event for the two $c r y$ integration mutants occurred. The excision frequencies of the MobSpc cassette in BMB0261::MobSpc (3 out of 100 colonies) and BMB0262::MobSpc (2 out of 100 colonies) were similar to that of BMB0260::MobSpc. Using PCR, we confirmed that the cry gene expression cassettes were integrated at the $a m y E$ site, together with the oriT fragment, in the unmarked BMB0261 and BMB0262 mutants (Fig. 6c, d). The PCR products were sequenced to confirm that BMB0261 and BMB0262 were properly constructed by integrating the 4646-bp cry $5 \mathrm{Ba}$ gene and the 3939-bp cry2Aa expression cassette at the amyE locus flanking the 24-bp oriT2/1 site.

Based on phase-contrast microscopy and scanning electron microscopy of the two cry integration mutants, the BMB0261 strains produced diamond-shaped crystals, and the BMB0262 strains produced round-shaped crystals (Fig. 6e; Additional file 1: Fig. S3A). Compared with the recombinant strains BMB171/cry5Ba-pHT304 and BMB171/cry2Aa-pHT304, in which the cry genes were cloned into the pHT304 vector, and the resulting plasmids were used to transform BMB171, the crystals produced by BMB0261 and BMB0262 were much smaller. This may be because the copy number of the plasmid vector pHT304 is much higher than a one-copy chromosome. Based on SDS-PAGE, protein expression is lower in BMB0261 and BMB0262 than the recombinant stains BMB171/cry5Ba-pHT304 and BMB171/cry2AapHT304, which supports this interpretation (Additional file 1: Fig. S3B). The development of the integration strains BMB0261 and BMB0262, results in bacterial production of insecticidal crystal proteins, especially for cry $5 \mathrm{Ba}$, which is silent in its original strain YBT-1518.

\section{Unmarked disruption of a seryl-AMP synthetase gene in zwittermicin A biosynthetic gene cluster}

To test whether this Mob/oriT recombination system could be used for the unmarked disruption in B. cereus, the $z m a J$ gene, which encodes seryl-AMP synthetase involved in (2S) amino malonyl-ACP formation in the zwittermicin A (ZmA) biosynthetic gene cluster [24] was selected as a deletion target. Plasmid pBMB0263 was constructed to disrupt $z m a J$ using pRec-mob-Ts (Fig. 7a). The unmarked integration mutants were generated following similar methods to those for amyE disruption and cry gene insertion. The unmarked integration mutants were confirmed by PCR using primers outside the homologous arms (Fig. 7b). The excision frequencies of the MobSpc cassette in BMB0263::MobSpc (3 out of 100 colonies) were similar to that of BMB0260::MobSpc. The PCR product was sequenced to confirm that BMB0263 was constructed by replacing a 1341-bp internal $z m a J$ gene fragment with a 36-bp segment containing the 24-bp oriT and two 6-bp restriction sites. As shown in Fig. 7c, using high resolution LC-MS to detect the production of $\mathrm{ZmA}$, the zmaJ deletion mutant BMB0263 
a

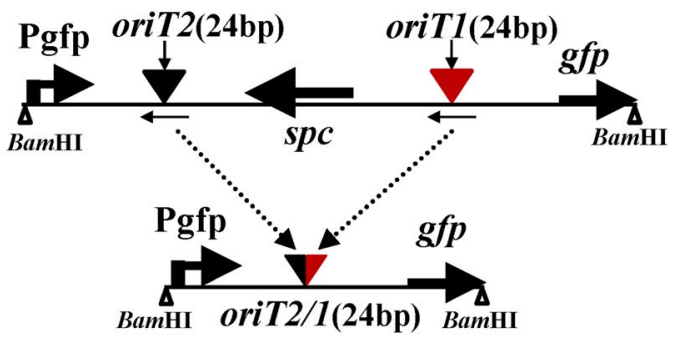

b

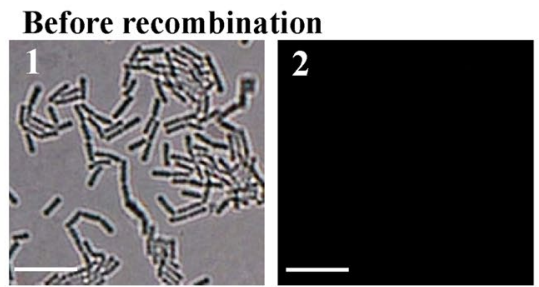

After recombination

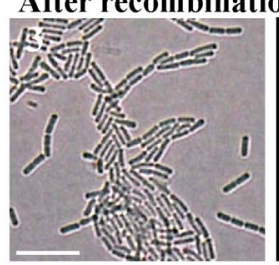

PC

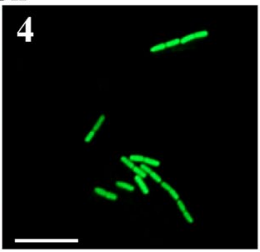

GFP c

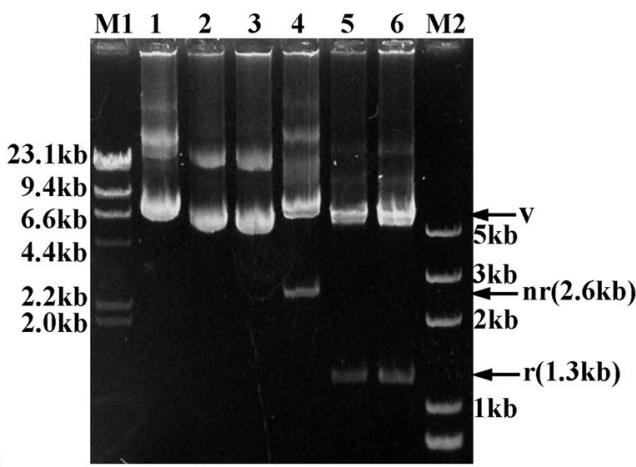

d

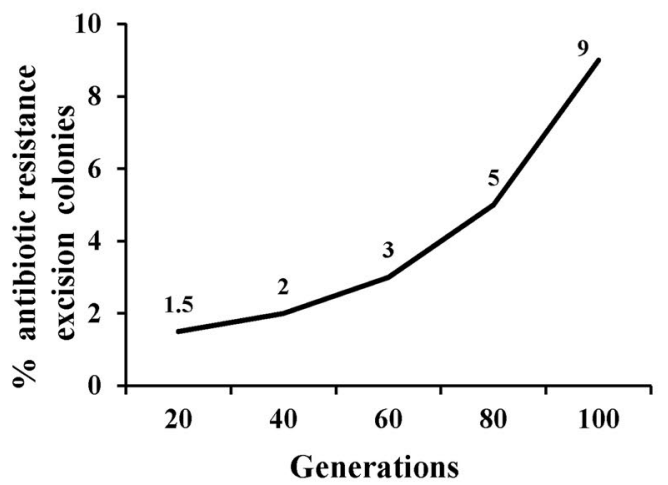

Fig. 3 Recombination frequency of pBMBTmini. a Structure of the recombination cassette and the resulting product after recombination. Arrows indicate the direction of transcription; spc spectinomycin-resistance gene, Pgfp the promoter of the kanamycin-resistance gene, gfp gene encoding green fluorescent protein. b Observation of BMB171 (pBMBTmini + pBMBmob1) before and after recombination. $P C$ phase-contrast microscopy; GFP fluorescence microscopy. Bar indicates $10 \mu \mathrm{m}$. c Restriction enzyme digestion of substrate plasmid before and after recombination; $M 1$ $\lambda$ DNA/HindIII marker; M2 Trans2 K Plus DNA marker.1, before recombination, substrate plasmid pBMBTmini; 2-3, from two single $\mathrm{Spc}^{\mathrm{S}}$ colonies after

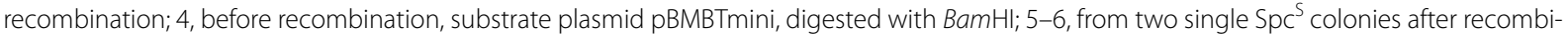
nation, digested with BamHl; $v$ vector, $6.5 \mathrm{~kb}$; $n$ r non-recombined cassette, $2.6 \mathrm{~kb}$. r recombined cassette, $1.3 \mathrm{~kb}$. $\mathbf{d}$ Increase of the percentage of antibiotic-resistance excision colonies over the number of generations

cannot produce ZmA, unlike UW85R. Thus, gene deletion via Mob-mediated recombination is feasible in $B$. cereus. In addition, complementation of $z m a J$ via pEMB0603-zmaJ to BMB0263 rescued the wild-type phenotype.

\section{Discussion}

Several B. thuringiensis and B. cereus strains have low electroporation efficiency or are not competent for transformation $[25,26]$, thus rendering DNA transfer by conjugation is the method of choice. To test the mobilization ability of Mob02281 into various B. thuringiensis and $B$. cereus strains, the previously constructed mobilizable plasmid pHTMob02281, which contains Mob02281 with oriT1, was used [19]. The successful transfer of pHTMob02281 into various $B$. thuringiensis and B. cereus strains was confirmed. Thus, pHTMob02281 can be used as a mobilizable shuttle vector for gene expression. We also confirmed its mobilization ability during the construction of an in-frame $z m a J$ deletion mutant in $B$. cereus UW85R. Therefore, this Mob02281/oriT system provides an alternative approach for genetic manipulations in these strains, which have rather low electroporation efficiencies.

Using this approach, the spectinomycin-resistant gene can be used to screen the Mob-Spc cassette integration mutants at the target locus. Antibiotics can be added to the medium to ensure that the excision of the cassette does not occur prematurely. Increasing the number of generations can increase the recombination frequency, and the excision of the Mob-Spc cassette occurs at the optimum growth temperature for B. thuringiensis and $B$. cereus strains.

We temporarily inserted an antibiotic resistance cassette flanked by pBMB0228 oriT sites oriented as direct repeats into a specific genomic region and subsequently removed it using the Mob/oriT system. The absence of the antibiotic resistance markers assures that cell physiology is not altered by known or unrecognized activities of resistance genes or antibiotic drugs. The cry $5 \mathrm{Ba}$ gene 


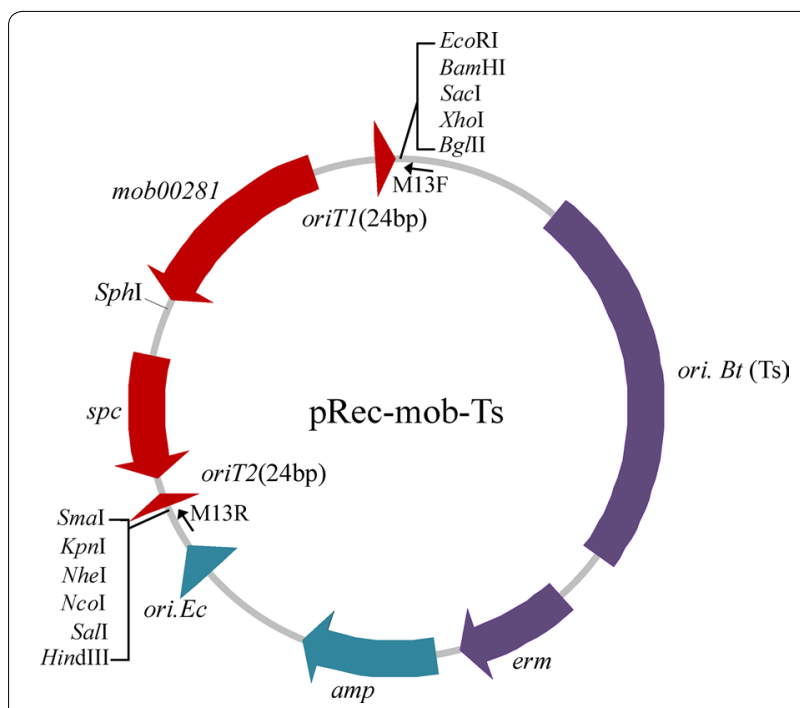

Fig. 4 Map of the temperature-sensitive-mobilizable recombination vector pRec-mob1-Ts. Arrows indicate the direction of transcription or replication. The Mob-Spc cassette is shown in red, including the spectinomycin-resistance gene (spc), the mob02281 gene, and the two mini-oriT sites (24-bp). The orientations of the replication of E. coli (ori.Ec) and the ampicillin-resistance gene ( $a m p$ ) are shown in light green; the orientations of the replication of $B$. thuringiensis and the erythromycin-resistance gene (erm) are shown in purple. The purple box flanking ori.Bt (TS) indicates that the replication orientation of the temperature-sensitive replication region of $B$. thuringiensis is not known

is a nematicidal protein gene, but it is silent in its original strain YBT-1518 [23], and cry2Aa cassette encodes proteins that form insecticidal crystal proteins active against lepidopteran and dipteran larvae [27]. Using this unmarked system, we constructed cry5Ba and cry $2 \mathrm{Aa}$ integration strains in $B$. thuringiensis BMB171. The two strains with cry integration can be used as genetically modify bacteria for the production the insecticidal crystal proteins because they avoid contamination and the risk of antibiotic resistance gene transfer to the environment. Although the protein expression is lower in the two strains with cry integration than the recombinant strains containing the cry genes maintained in a plasmid vector because the copy number of a one-copy chromosome is much less than the plasmid vector, the protein production can be improved by replacing the original promoter with a strong promoter to express the cry genes. In the long term, the cry gene integrated in the chromosome is much stable than maintained in a plasmid vector. Thus, Mob/oriT system provides an efficient marker-free approach for constructing genetically modify bacteria.

The zmaJ gene within the operon of the ZmA biosynthetic gene cluster was selected as a target for deletion. Complementation of $z m a J$ to the $z m a J$ deletion strain rescued the wild-type phenotype. This result suggests that the 36-bp DNA remnants after Mob02281-mediated recombination did not affect the expression of other genes in the operon.

In all current site-specific recombination systems, including the FLP/FRT system [6-8], Cre/loxP system $[9,10]$, Xer/dif system [11, 12], and Relaxase/oriT system $[16,28]$, a single recombination site remains within the target region, and undesired recombination might occur between recombination sites that accumulate after multiple rounds of gene disruption. In the Cre/loxP system, Cre recombinase activity was limited to produce defined recombination events in strains containing multiple loxP sites [10]. The combination of these systems may resolve these issues when multiple genes are manipulated in a target strain. In this study, to minimize undesired recombination using the Mob02281/oriT system, we used the original promoter, rather than an inducible strong promoter, to express Mob02281.

A TnpI-mediated recombination system has been used to construct genetically engineered $B$. thuringiensis strains. The cry $1 C$ gene is introduced and maintained in a plasmid vector whose antibiotic resistance genes are flanked by two internal resolution sites. The antibiotic resistance genes of the plasmid are then eliminated by TnpI-mediated recombination between the two internal resolution sites [29]. However, the long-term stability of cry $1 C$ expression is an issue. Moreover, this method has not been assessed with respect to gene deletion. In B. anthracis, the Cre/loxP system can be used to efficiently inactivate individual genes and facilitate large deletions [10]. In this approach, two temperature-sensitive plasmids are electroporated into the target strain. One contains a resistance cassette flanked by loxP sites and is used to temporarily insert the resistance gene into a selected genomic region based on homologous sequences. The other expresses the Cre recombinase and is used to remove the resistance gene after recombination between loxP sites [10]. This process cannot be applied to some wild-type $B$. cereus group strains in which electroporation are inefficient.

We also tested the recombination ability of the Mob02281/oriT system in other bacterial taxa; its inefficiency in $E$. coli indicated that it might have specific host requirements. However, in addition to Mob02281, certain relaxases can catalyze site-specific recombination between two oriT copies, including NikB of plasmid R64 [16], TrwC of plasmid R388 [17], MobA of plasmid R1162 [18] from Gram-negative bacteria, MobB and MobE of plasmid pAM $\alpha 1$ [30], and the Mobs of plasmid pE194 and pT181 [31] from Gram-positive bacteria. Therefore, future studies should examine various relaxase/oriT elements to extend the applications of this alternative recombination system for unmarked genetic 


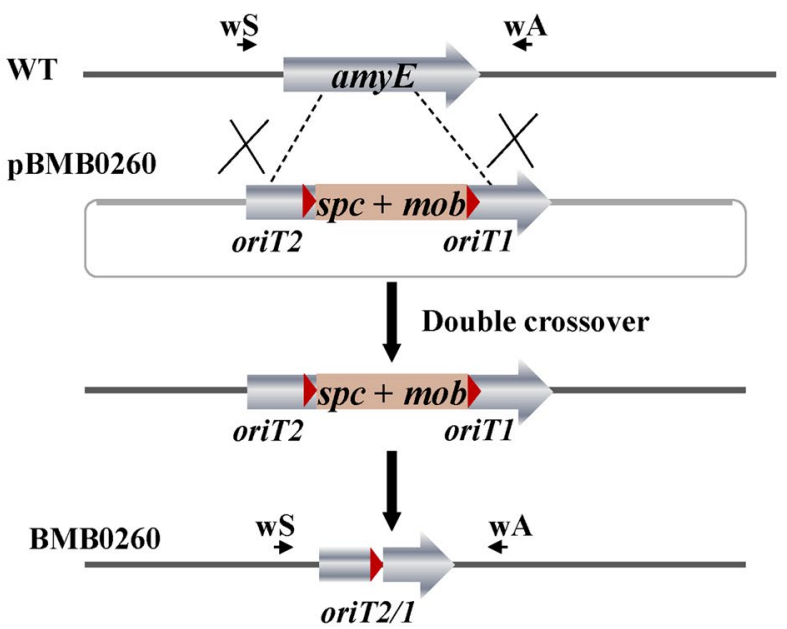

$\mathbf{b}$

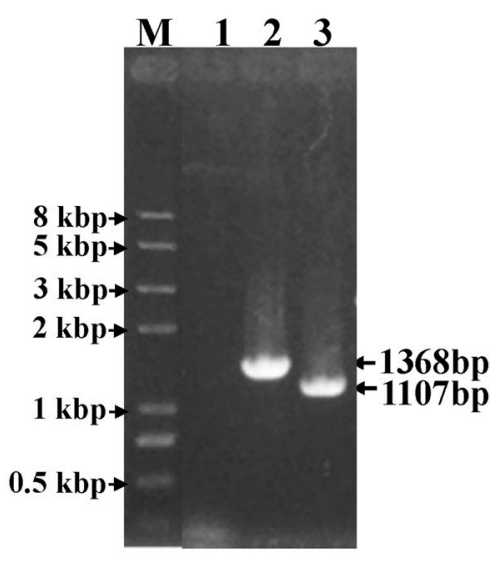

c

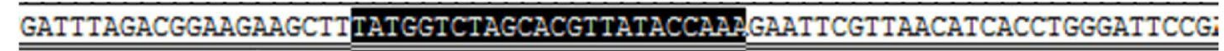
GATTTAGACGGAAG

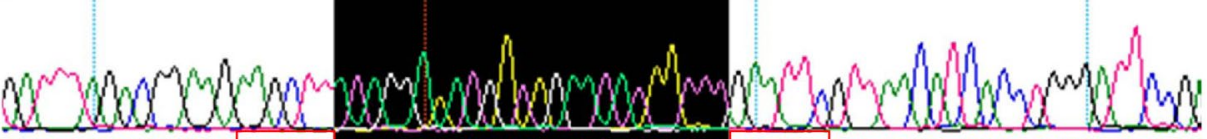
GATTTAGACGGAAGAGCIITATGGICTAGCACGTTATACCAAP GAATIDGTIAACATCACCIGGGATTCCG: HindIII $\operatorname{oriT2/1(24\mathrm {bp})}$

upstream homologous arms

downstream homologous arms

Fig. 5 Design and confirmation of amyE disruption in B. thuringiensis BMB171. a Depiction of amyE disruption. b PCR detection with primers amyEwS and amyE-wA. DNA templates were from: 1 pBMB0260 (negative control); 2 BMB171; 3 amyE gene-disruption strain BMB0260; M Trans2 K Plus II DNA marker; c Sequence analysis of the deletion region of BMB0260

manipulations to a wider range of taxa, including both Gram-negative and Gram-positive bacteria.

\section{Conclusions}

The Mob/oriT mobilizable recombination system can be exploited for unmarked genetic manipulation and construction of genetically modify bacteria in $B$. thuringiensis and B. cereus. Using this system, the recombination vectors were constructed and the application was tested by in-frame deletion in B. thuringiensis and B. cereus and insertion the interested gene into chromosome in $B$. thuringiensis. We also confirmed the putative amylase gene can be used as integration site for gene expression in B. thuringiensis and B. cereus.

\section{Methods}

\section{Bacterial strains, plasmids, growth conditions, and DNA} manipulations

The strains and plasmids used in this study and their sources are listed in Table 2. E. coli was cultured in LB medium at $37{ }^{\circ} \mathrm{C}$, and all B. thuringiensis and B. cereus strains were cultured in LB medium at $28{ }^{\circ} \mathrm{C}$. Spectinomycin, tetracycline, erythromycin, kanamycin, ampicillin, rifampin, and streptomycin were added to the media at final concentrations of 100, 10, 25, 50, 100, 100, and $100 \mu \mathrm{g} / \mathrm{mL}$, respectively. All regular DNA manipulations were carried out following standard methods [32]. All recombinant plasmids were constructed in $E$. coli, and plasmids were extracted from $E$. coli according to the methods of Sambrook and Russell [32]. PCR products were confirmed by DNA sequencing. Primers used for PCR amplification are listed in Table 3.

\section{Transformation of $E$. coli and B. thuringiensis}

E. coli transformation was carried out using $\mathrm{CaCl}_{2}$ treated competent cells, as described by Sambrook [32]. $B$. thuringiensis transformation was performed by electroporation with the Bio-Rad Gene Pulser (Hercules, CA, USA) with previously described settings [25].

\section{Plasmid construction}

To decrease the influence of DNA remnants after Mob02281-mediated recombination, we identified the minimal oriT sequence required for Mob02281-mediated recombination. Using the previous recombination system [19], we performed a series of deletions in one or both 
a

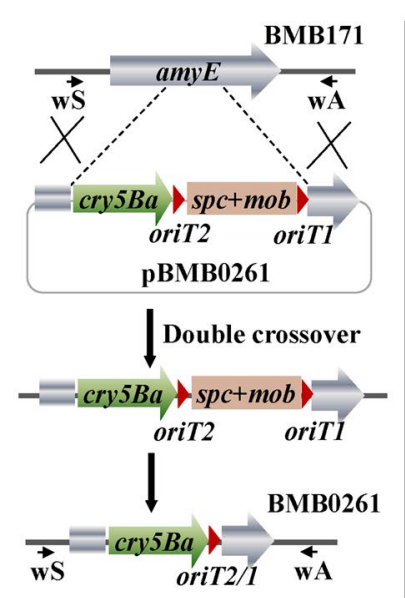

e
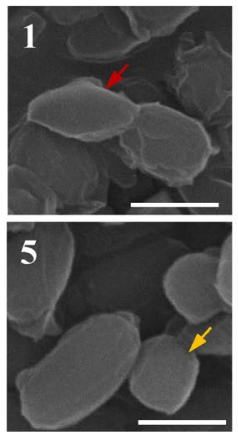

b

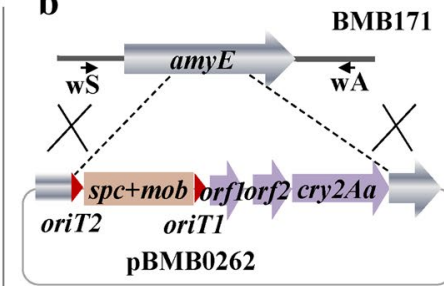

| Double crossover

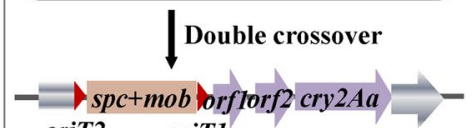

oriT2 oriT1

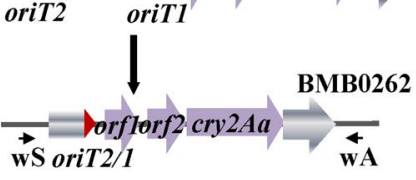

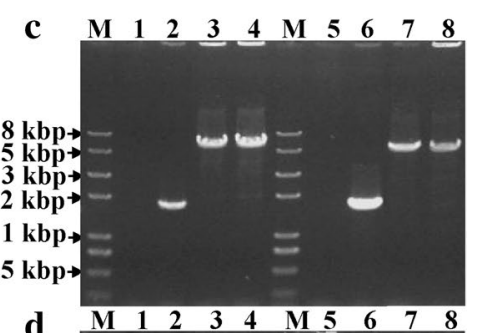
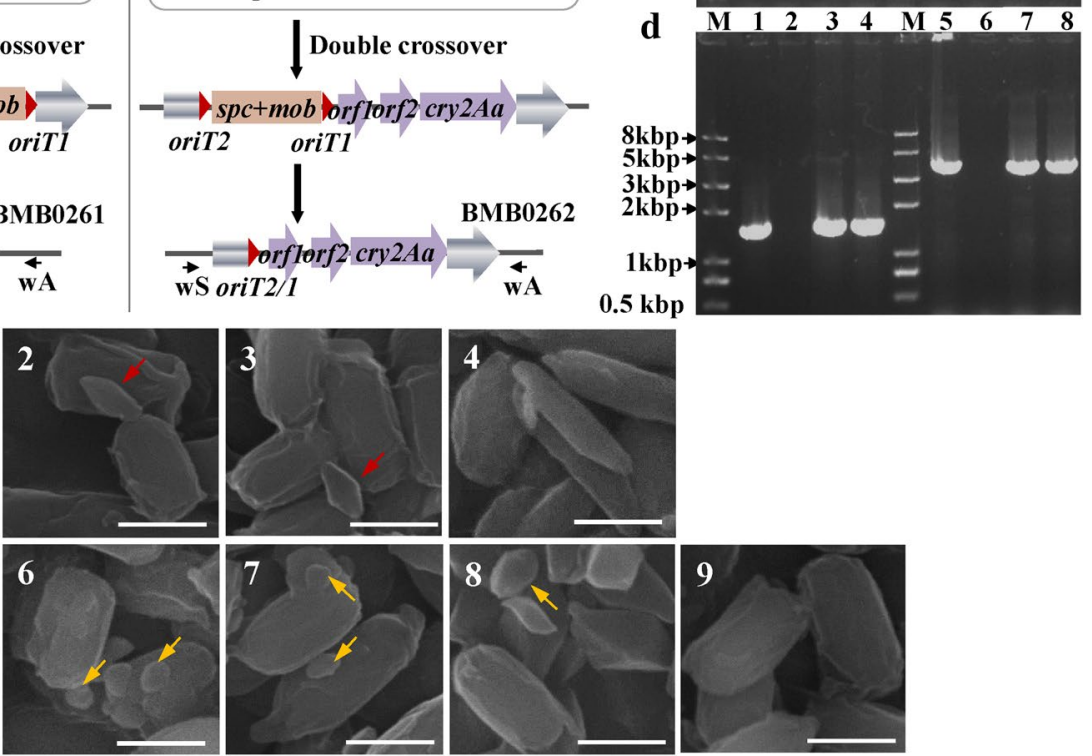

Fig. 6 Construction of crystal protein gene insertion mutants BMB0261 and BMB0262. Depiction of cry5Ba (a) and cry2Aa (b) insertion. PCR detection with primers amyE-wS and amyE-wA (c) and 5B-S and 5B-A (lanes 1-4) or 2A-S and 2A-A (lanes 5-8) (d); DNA templates were from: 1 , pBMB0261 (negative control); 2 and 6, BMB171; 3-4, mutant BMB0261; 5, pBMB0262 (negative control); 7-8, mutant BMB0262; M Trans2 K Plus II DNA marker. e Scanning electron microscopy image of parasporal crystals in unmarked cry integrate mutants. 1, BMB171/cry5Ba-pHT304; 2-3, BMB0261; 4, YBT-1518; 5, BMB171/cry2Aa-pHT304; 6-7, BMB0262; 8, CT-43; 9, BMB171. The arrows indicate the diamond-shaped crystals encoded by cry5Ba and the round-shaped crystals encoded by cryzAa. Bar indicates $1 \mu \mathrm{m}$

oriTs (352-bp oriT1 and 370-bp oriT2) of the substrate plasmid pBMBT10.

The substrate plasmids pBMBT11, pBMBT12, pBMBT13, pBMBT14, pBMBT15, pBMBT16 and pBMBTmini was constructed with a similar organization to that of pBMBT10 [19]. The truncated oriT1 fragments containing bp 1-352, 65-282 and 188-282 were respectively amplified from pBMB0228 using the primer pair oriT1-1S/oriT1-352A, oriT1-65S/oriT1282A, oriT1-188S/oriT1-282A. The truncated oriT2 fragments containing bp $1-370,156-370$ and $273-370$ were respectively amplified from pBMB0228 using the primer pair oriT2-1S/oriT2-370A, oriT2-156S/oriT2370A and oriT2-273S/oriT2-370A. Plasmids pBMBT11, pBMBT12 and pBMBT13 were constructed by remaining the bp 1-352 oriT1 unchanged, and respectively containing the bp 156-370, 273-370, and 273-297 of oriT2. Plasmids pBMBT14, pBMBT15 and pBMBT16 were constructed by remaining the bp 1-370 of oriT2 unchanged, and respectively containing the bp 65-282, 188-282 and 258-282 of oriT1. Plasmid pBMBTmini contains 24-bp core oriT1 and 24-bp core oriT2 sequences. The two 24-bp core oriT sequences in the same orientation flanking a spectinomycin-resistance marker were amplified with the primer pair oriT1-24-S/oriT1-24-A using pBMBT10 as a template. Other construction steps of these substrate plasmids were similar to those described previously for the construction of pBMBT10 [19].

The mobilizable module, which comprised the mob02281 gene and a 352-bp oriT1 region overlapping its promoter, was amplified from pBMB0228 using the primer pair MS/MA, and the spectinomycin-resistance gene (a selection marker) was amplified from pIC333 [33] using the primer pair SS/SA. The 1.9- and 1.3-kb PCR products were digested with EcoRI/SphI and SphI/SalI, respectively, and cloned into the EcoRI/SalI sites of the Ts vector pHT304Ts, generating the Ts mobilizable sitespecific recombination vector pRec-mob-Ts. Multiple restriction sites were introduced into $\mathrm{pRec}$-mob-Ts with primers by PCR amplification. 
a

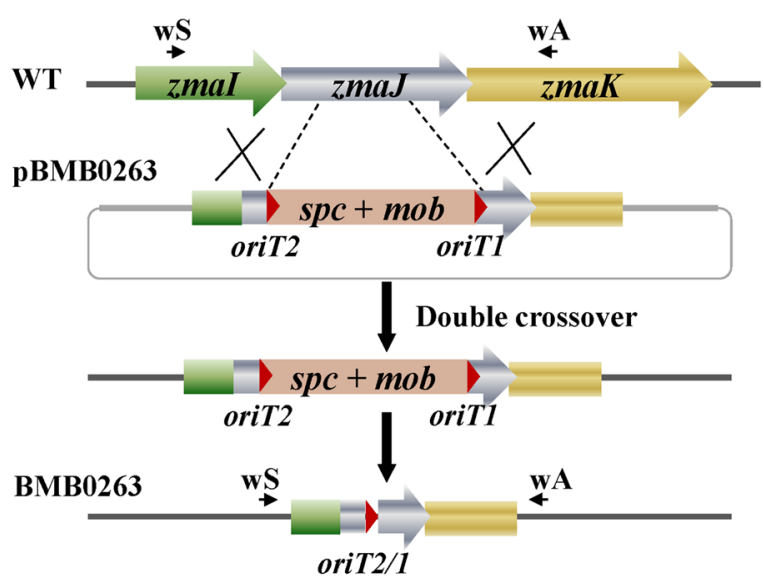

c

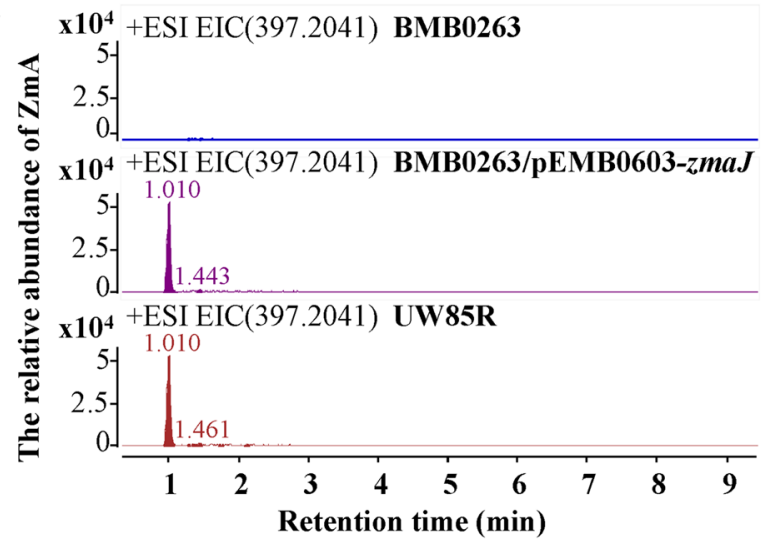

b

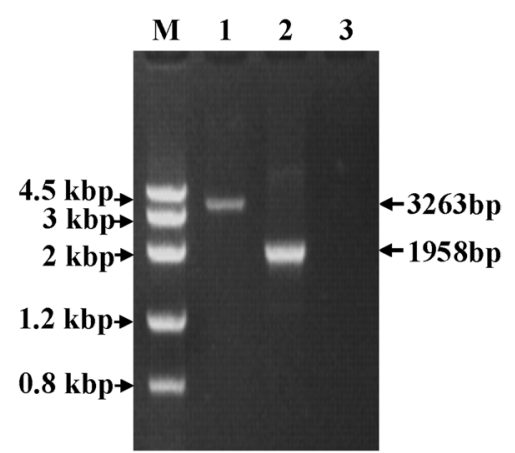

Fig. 7 Design and confirmation of zmaJ disruption in B. cereus UW85R. a Depiction of zmaJ disruption. b PCR detection with primers zmaJ-wS and zmaJ-wA. DNA templates were from: 1, UW85R; 2, zmaJ gene-disrupted strain BMB0263; 3, pBMB0263 (negative control); M, DNA Marker III. c LC-MS detection of a molecular weight corresponding to ZmA in the culture supernatants. The molecular weight corresponding to ZmA is indicated by a rectangular frame

To generate the complementation plasmid pEMB0603$z m a J$, the promoter of kanamycin resistance gene was amplified from pBMBT10 using the primer pair pKan-S/ pKan-zmaJ-A, and the coding region of the $z m a J$ gene was amplified from $B$. cereus UW85 genomic DNA using the primer pair pKan-zmaJ-S/zmaJ-A. Using overlap PCR with the primer pair pKan-S/zmaJ-A, a PCR fragment containing the coding region of $z m a J$ and a promoter of the kanamycin resistance gene was generated. Then, this 2.1-kb PCR fragment was digested with EcoRI/SalI and inserted into the same sites of the shuttle vector pEMB0603, resulting in plasmid pEMB0603-zmaJ.

\section{Construction of unmarked mutant strains BMB0260, BMB0261, BMB0262, and BMB0263}

To generate the $a m y E$ mutant strain $\mathrm{BMB0260 \text {,two }}$ primer pairs, amyE-up-S/amyE-up-A and amyE-down-S/ amyE-down-A, were used to amplify the upstream and downstream homologous arms of the $a m y E$ gene from BMB171 genomic DNA. The 566- and 529-bp fragments were digested with EcoRI/BamHI and SalI/HindIII, respectively, and cloned into the corresponding sites of pRec-mob-Ts one by one, generating pBMB0260.

To demonstrate that the Mob/oriT recombination system could be used to introduce a gene of interest, plasmids pBMB0261 and pBMB0262, containing cry5Ba and $\operatorname{cry} 2 \mathrm{Aa}$ (together referred to as $c r y$ genes), were constructed based on the integrative vector pBMB0260. The $c r y 5 \mathrm{Ba}$ gene was digested with HindIII from its plasmid pBMB0215 [23] and inserted into the HindIII site of pBMB0260. The cry2Aa expression cassette containing orf1, orf 2 and $\operatorname{cry} 2 \mathrm{Aa}$ [34] was amplified using the primer pair 2A-S/2A-A from B. thuringiensis CT-43, digested with EcoRI, and inserted into the EcoRI site of pBMB0260. The resulting plasmids pBMB0261 and pBMB0262 contains cry genes and could be integrated into the amyE locus via the $a m y E$ homologous arms (Fig. 6a, b).

To generate the $z m a J$ mutant strain BMB0263, two primer pairs, zmaJ-up-S/zmaJ-up-A and zmaJ-down-S/ 
Table 2 Bacterial strains and plasmids used in this study

\begin{tabular}{|c|c|c|}
\hline Strains or plasmids & Description $^{a}$ & Reference \\
\hline \multicolumn{3}{|c|}{ Bacillus thuringiensis strains } \\
\hline AW48 & Derivative of wild-type isolate HD73 cured of pHT73, containing pAW63::Tn5401, Tet ${ }^{r}$ & {$[36]$} \\
\hline BMB0260 & Mutant of strain BMB171 in which the amyE gene is interrupted by in-frame deletion & This study \\
\hline BMB0261 & Mutant of strain BMB171 in which the cry5Ba gene is inserted in the amyE locus & This study \\
\hline BMB0262 & Mutant of strain BMB171 in which the cry2Aa expression cassette is inserted in the amyE locus & This study \\
\hline BMB171 & Acrystalliferous mutant of $B$. thuringiensis & {$[39]$} \\
\hline BMB171Str & Streptomycin spontaneous mutant strain of BMB171, Str ${ }^{r}$ & This study \\
\hline YBT-1520Str & Streptomycin spontaneous mutant strain of B. thuringiensis serovar kurstaki YBT-1520, Str ${ }^{r}$ & This study \\
\hline $\mathrm{CT}-43$ & No flagellum, containing cry2Aa expression cassette and thuringiensin synthesis cluster & {$[40]$} \\
\hline CT-43::Kan & $\begin{array}{l}\text { Mutant of strain CT-43 in which the thuE gene is interrupted by the kanamycin-coding gene, also named } \\
\text { BMB0545, } \text { Kan }^{r}\end{array}$ & {$[41]$} \\
\hline \multicolumn{3}{|l|}{ Bacillus cereus strains } \\
\hline ATCC 10987Str & Streptomycin spontaneous mutant strain of B. cereus ATCC 10987, Str $^{r}$ & This study \\
\hline UW85 & Wild type B. cereus strain, ZmA producing & {$[24]$} \\
\hline UW85R & Rifampin spontaneous mutant resistance strain of B. cereus UW85, Rif ${ }^{\top}$ & This study \\
\hline BMB0263 & Mutant of strain UW85R in which the zmaJ gene is interrupted by in-frame deletion & This study \\
\hline \multicolumn{3}{|l|}{ Escherichia coli strains } \\
\hline $\mathrm{DH} 5 \mathrm{a}$ & 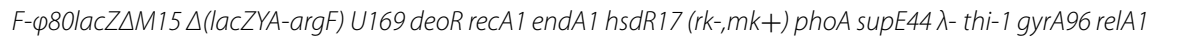 & Invitrogen \\
\hline \multicolumn{3}{|l|}{ Plasmids } \\
\hline pAW63::Tn5401 & $\begin{array}{l}\text { A self-transmissible plasmid generated by insertion of the tetracycline-resistance transposon } \\
\text { Tn5401 in pAW63, } \text { Tet }^{r}\end{array}$ & {$[36]$} \\
\hline pBMB0215 & A recombinant plasmid harboring a 4.6-kb Hindlll fragment including cry5Ba & {$[23]$} \\
\hline pBMB0228 & 17,706-bp endogenous plasmid in strain YBT-1518 & {$[19,42]$} \\
\hline pBMBmob1 & pEMB0603 containing a 1.8-kb BamHI/Sphl fragment carrying mob02281 & {$[19]$} \\
\hline pBMBT10 & pHT315 containing the recombination cassette with bp 1-352 of oriT1 and bp 1-370 of oriT2 & {$[19]$} \\
\hline pBMBT11 & pHT315 containing the recombination cassette with bp 1-352 of oriT1 and bp 156-370 of oriT2 & This study \\
\hline pBMBT12 & pHT315 containing the recombination cassette with bp 1-352 of oriT1 and bp 273-370 of oriT2 & This study \\
\hline pBMBT13 & pHT315 containing the recombination cassette with bp 1-352 of oriT1 and bp 273-297 of oriT2 & This study \\
\hline pBMBT14 & pHT315 containing the recombination cassette with bp 65-282 of oriT1 and bp 1-370 of oriT2 & This study \\
\hline pBMBT15 & pHT315 containing the recombination cassette with bp 188-282 of oriT1 and bp 1-370 of oriT2 & This study \\
\hline pBMBT16 & pHT315 containing the recombination cassette with bp 258-282 of oriT1 and bp 1-370 of oriT2 & This study \\
\hline pBMBTmini & $\begin{array}{l}\text { pHT315 containing the recombination cassette as in PBMBT10 [19] with the 24-bp core sequence of } \\
\text { oriT1 and 24-bp core sequence of oriT2 }\end{array}$ & This study \\
\hline pEMB0603 & E. coli and B. thuringiensis shuttle vector, Amp ${ }^{r}, \mathrm{Kan}^{\mathrm{r}}$ & {$[35]$} \\
\hline pEMB0603-zmaJ & $\begin{array}{l}\text { pEMB0603 containing a } 2.1 \mathrm{~kb} \text { EcoRI/Sall fragment carrying the coding sequence of the seryl-AMP } \\
\text { synthetase gene ZmaJ with the promoter of kanamycin resistance gene }\end{array}$ & This study \\
\hline pHT315 & E. coli and B. thuringiensis shuttle vector, Amp ${ }^{r}$, Erm $^{r}$ & {$[43]$} \\
\hline pHT304 & E. coli and B. thuringiensis shuttle vector, Amp ${ }^{r}, \mathrm{Erm}^{r}$ & {$[43]$} \\
\hline pHT304Ts & $\begin{array}{l}\text { E. coli and B. thuringiensis shuttle vector, containing a temperature-sensitive replicon in B. thuringiensis, } \\
\text { Ampr }^{r}, \text { Erm }^{r}\end{array}$ & {$[35]$} \\
\hline pHTMob02281 & pHT304 containing a 1.8-kb HindIII fragment carrying mob02281 and oriT1 & This study \\
\hline plC333 & An efficient mini-Tnl0 delivery vector in B. subtilis, Spc ${ }^{r}$, Erm $^{r}$ & {$[33]$} \\
\hline pRec-mob1-Ts & $\begin{array}{l}\text { pHT304Ts containing the Mob-Spc cassette and 24-bp core oriT recombination sequence, } \\
\text { Ampr }{ }^{r}, \mathrm{Erm}^{r}, \mathrm{Spc}^{r}\end{array}$ & This study \\
\hline
\end{tabular}

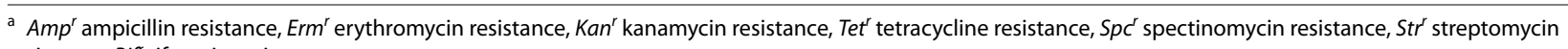
resistance, $R$ if rifampin resistance 
Table 3 Primers used in this study

\begin{tabular}{|c|c|c|}
\hline Primers & Sequence $^{\mathrm{a}}\left(5^{\prime}-3^{\prime}\right)$ & Construct(s) or use \\
\hline oriT1-1S & CGAGAATTCGTAAGCGGAACGAAGTG & PCR amplification of bp 1-352,65-282 and 188-282 of oriT1 \\
\hline oriT1-65S & CGAGAATTCTGTTAAAACGGTTACCGTTTTG & \\
\hline oriT1-352A & CAGGTCGACCTACATGAGCCACGATCAATC & \\
\hline oriT1-282A & CAGGTCGACTTTGGTCTAGCACGTTATACC & \\
\hline oriT1-188A & CAGGTCGACGACTTTGTCCGAACCTACAAG & \\
\hline oriT2-1S & TATGCATGCCAAAGGTACCGGACCGAACC & PCR amplification of bp 1-370, 156-370 and 273-370 of oriT2 \\
\hline oriT2-370A & CAGAAGCTTGAACGTGAGCCATGAGCGAG & \\
\hline oriT2-156S & TATGCATGCGAAGGGGGTCAAGGGGAATT & \\
\hline oriT2-273S & TATGCATGCATTTGGTATAACGTGCTAGAC & \\
\hline oriT1-24-S & TATGAATTCTTTGGTATAACGTGCTAGACCAAATCTGACGCTCAGTGG & pBMBT13, pBMBT16 and pBMBTmini \\
\hline oriT2-24-A & CCCAAGCTTTATGGTCTAGCACGTTATACCAAAAAATGTCACTAATATT & \\
\hline MS & $\begin{array}{l}\text { GAATTCGGATCCGAGCTCCTCGAGAGATCTTTTGGTCTAGCACG } \\
\text { TTATACCAAAGTGTGTTCTTAATATTGTATG }\end{array}$ & pRec-mob1-Ts \\
\hline MA & CGCGTCGACGCATGCGATTACAACAAGTCTATTCAG & \\
\hline SS & CGCGCATGCTCTGACGCTCAGTGGAAC & \\
\hline SA & $\begin{array}{l}\text { GTCGACCCATGGGCTAGCGGTACCCGGGTTTGGTATAACGTGC } \\
\text { TAGACCATACTATGCAAGGAACAATTTC }\end{array}$ & \\
\hline amyE-up-S & CGCGAATTCATGCGTTTCTCTCGGATAATTGG & BMB0260, BMB0261 and BMB0262 \\
\hline amyE-up-A & CTCGGATCCCTTCCGTCTAAATCAACTTC & \\
\hline amyE-down-S & CGCGTCGACGTTAACATCACCTGGGATTC & \\
\hline amyE-down-A & GCCAAGCTTTCATCTACGCTTCCTTTTAACTG & \\
\hline amyE-wS & GTGCCAATCCTGGTGTCATC & \\
\hline amyE-wA & GAAAATCCTCTTCCTAACCTG & \\
\hline $5 B-S$ & GGGTATACAAGAAGGTTGGG & cry5Ba detection \\
\hline $5 B-A$ & CGTTTTCTGGTACAAGTTCC & \\
\hline $2 A-S$ & GCCGAATTCGATGTTGATTCTTAGAGCAATG & BMB0262 \\
\hline $2 \mathrm{~A}-\mathrm{A}$ & GGCGAATTCGGTTAACTTGAAATGATTTCTCC & \\
\hline zmaJ-up-S & CGGGAATTCCATTGGTAGACCAGGATTTGC & BMB0263 \\
\hline zmaJ-up-A & CATGGATCCTCCACCTTCACGTAAAAGACG & \\
\hline zmaJ-down-S & CGGGGTACCGTACTTGCCTTATTAGGTTCCG & \\
\hline zmaJ-down-A & CACGTCGACACTTACACGAAGTGGTGGTG & \\
\hline zmaJ-wS & CAGCTGCTGATGTGTTTTTGG & \\
\hline zmaJ-wA & CTCAACTACCATAATAGTGTC & \\
\hline pKan-S & CCGGAATTCCATTTGAGGTGATAGGTAAG & pEMB0603-zmaJ \\
\hline pKan-zmaJ-A & $\begin{array}{l}\text { TATACATGTACTCATTTTAATAACCTCCTTTCTCTAGACCCCAA } \\
\text { GAAGCTAATTATAAC }\end{array}$ & \\
\hline pKan-zmaJ-S & $\begin{array}{l}\text { ATTAGCTTCTTGGGGTCTAGAGAAAGGAGGTTATTAAAATGA } \\
\text { GTACATGTATACAAAAG }\end{array}$ & \\
\hline zmaJ-A & ACGCGTCGACTTATGAATCTTTACTGCTTTC & \\
\hline
\end{tabular}

a Restriction sites included in oligonucleotide sequences are italicized

zmaJ-down-A, were used to amplify the upstream and downstream homologous arms of the $z m a J$ gene from $B$. cereus UW85 genomic DNA. The 675- and 940-bp fragments were digested with EcoRI/BamHI and KpnI/SalI, respectively, and cloned into the corresponding sites of pRec-mob-Ts one by one, generating pBMB0263 (Fig. 7a).

Using spectinomycin, mutants containing the MobSpc cassette were selected following a general method used for B. thuringiensis gene knockout [35]. Briefly, the Ts plasmids pBMB0260, pBMB0261, pBMB0262, and $\mathrm{pBMB0263}$ were used to transform $B$. thuringiensis BMB171 by electroporation. The transformant BMB171/ pBMB0263 was used as the donor strain, AW48 carrying the conjugative plasmid pAW63:: $\operatorname{Tn} 5401$ was used as the helper strain, and B. cereus UW85R was used as the recipient strain. Triparental mating yielded the transconjugant UW85R/pBMB0263. The correct colonies BMB171/pBMB0260, BMB171/pBMB0261, BMB171/ 
pBMB0262, and B. cereus UW85R/pBMB0263 were cultivated in LB medium containing spectinomycin $(40 \mu \mathrm{g} /$ $\mathrm{mL}$ ) at $28{ }^{\circ} \mathrm{C}$ for $8 \mathrm{~h}$. These strains were incubated at $42{ }^{\circ} \mathrm{C}$ for 4 days to generate the Mob-Spc cassette integrated mutants and eliminate unintegrated Ts plasmids.

The expected mutant strains, which were resistant to spectinomycin $(100 \mu \mathrm{g} / \mathrm{mL})$, but sensitive to erythromycin $(25 \mu \mathrm{g} / \mathrm{mL})$, were collected and confirmed by PCR using appropriate primers and sequencing. Finally, the mutant strains BMB0260::Mob-Spc, BMB0261::MobSpc, BMB0262::Mob-Spc and BMB0263::Mob-Spc were obtained.

The mutant strains with Mob-Spc cassette integration were cultivated at $28{ }^{\circ} \mathrm{C}$ for $24 \mathrm{~h}$ to excise the MobSpc cassette via recombination mediated by Mob02281, which is encoded by the Mob-Spc cassette. To increase the population of mutant strains from which the MobSpc cassette was excised, bacterial suspensions were diluted 1000-fold in fresh LB liquid medium every $8 \mathrm{~h}$. The expected unmarked mutant strains, which were sensitive to spectinomycin $(100 \mu \mathrm{g} / \mathrm{mL})$, were collected and confirmed by PCR using primers outside the homologous arms and sequenced. Finally, the expected mutant strains BMB0260, BMB0261, ВMB0262, and BMB0263 were obtained.

\section{Plasmid transfer}

To test the mobilization ability of Mob02281 into various $B$. thuringiensis and B. cereus group strains, the previously constructed mobilizable plasmid pHTMob02281, which contains Mob02281 with oriT1, was used [19]. To facilitate phenotypic observations of DNA that is transferred to the recipient strains, the plasmid cry6AapHTMob02281, which contains cry6Aa as a reporter, was constructed and used to transform $B$. thuringiensis $\mathrm{BMB} 171$. As recipient strains, we used $B$. thuringiensis BMB171Str, B. thuringiensis CT-43::Kan, and B. thuringiensis serovar kurstaki YBT-1520Str, as well as the closely related species $B$. cereus strains UW85R and ATCC 10987Str. It should be noted that B. thuringiensis CT-43::Kan and YBT-1520Str have rather low electroporation efficiencies [25].

Conjugation assays were conducted following the protocols described by Andrup et al. [36]. In brief, overnight cultures of donor, recipient, and helper strains were incubated at $28^{\circ} \mathrm{C}$ in LB medium with appropriate antibiotics. Equal quantities of donor, recipient, and helper cells in logarithmic growth were mixed and incubated in $7 \mathrm{~mL}$ of pre-warmed LB medium, without antibiotics, at $28{ }^{\circ} \mathrm{C}$ with moderate shaking (180 rpm). After mating proceeded for $3 \mathrm{~h}$, appropriate dilutions were plated on selective medium to determine the number of transconjugants. Control donors, recipients, and helper strains were grown separately and tested in parallel. Transfer frequencies were calculated as the ratio of transconjugants to donor cells. Each experiment was performed in triplicate.

\section{Recombination assays}

Recombination assays were conducted following previously described methods [19]. To test the efficiency of Mob02281-mediated recombination, the substrate plasmids pBMBT11, pBMBT12, pBMBT13, pBMBT14, pBMBT15, pBMBT16 and pBMBTmini was respectively introduced to BMB171 containing the helper plasmid pBMBmob1. The resulting colonies were grown with erythromycin and kanamycin and plated on erythromycin and kanamycin plates by dilution, and the resulting single colonies were transferred onto spectinomycincontaining plates after 20,40,60, 80, and 100 generations. Every $8 \mathrm{~h}$ (approximately 10 generations) [29], bacterial suspensions were diluted 1000 -fold in $10 \mathrm{~mL}$ of fresh LB liquid medium. Recombination frequency was estimated as the number of spectinomycin-sensitive colonies. Each experiment was performed in triplicate.

\section{Microscopic observations}

For observations of the number of fluorescent cells under phase-contrast and fluorescence microscopy, all B. thuringiensis strains were cultivated in LB liquid medium at $28^{\circ} \mathrm{C}$. The morphology of the strains was observed with an Olympus photomicroscope (Tokyo, Japan).

To observe the production of parasporal crystals under phase-contrast microscopy, all strains were sporulated using a previously described method [37]. Briefly, strains were cultured in ICPM liquid medium (0.6\% peptone, $0.5 \%$ glucose, $0.1 \% \mathrm{CaCO}_{3}, 0.05 \% \mathrm{MgSO}_{4}$, and $0.05 \%$ $\left.\mathrm{KH}_{2} \mathrm{PO}_{4}(\mathrm{pH} 7.0)\right)$ for $36 \mathrm{~h}$ at $28^{\circ} \mathrm{C}$. The spores and crystals were collected and washed three times with $1 \mathrm{M} \mathrm{NaCl}$ solution and three times with water [37]. These samples were also used to detect crystal protein expression by sodium dodecyl sulfate polyacrylamide gel electrophoresis (SDS-PAGE). Under phase-contrast microscopy, spores are phase-bright, and crystals are phase-dark [35]. For scanning electron microscopy with a Quanta200 (FEI, Hillsboro, OR, USA), the lysed cell samples were treated following the methods described by Shao et al. [37].

\section{Detection of zwittermicin A ( $\mathrm{ZmA})$ by liquid chromatography mass spectrometry}

High-resolution liquid chromatography mass spectrometry (LC-MS) was performed using an Agilent 1260 LC device (Agilent, USA) equipped with a $\mathrm{C}_{18}$ reverse-phase column $(100 \times 1.8 \mathrm{~mm}$; particle size, $3.5 \mu \mathrm{m})$. The mobile phase consisted of chromatography grade water containing $0.1 \%$ formic acid in pump B and chromatography 
grade acetonitrile in pump A. The $1 \mathrm{uL}$ of 36 -h culture supernatants of UW85R and ZmA mutants were loaded. The elution step was performed under an acetonitrile concentration of $5 \%$ for $5 \mathrm{~min}$ at a flow rate of $0.3 \mathrm{~mL} /$ $\mathrm{min}$. The diode array detection was performed at $210 \mathrm{~nm}$. MS was performed using the Q-TOF(quadrupole-time of flight) MS G6540A system (Agilent) equipped with a dual-source electrospray ionization (ESI) ion source operated in positive-ion mode. Calibration was performed with standard references of mass 121.0509 and 922.0098. The source parameters were as follows: gas temperature of $350{ }^{\circ} \mathrm{C}$, gas flow rate of $8 \mathrm{~L} / \mathrm{min}$, and nebulizer stress of $35 \mathrm{lb} / \mathrm{in}^{2}$ (gauge). The capillary, fragmenter, skimmer, and octopole radio frequency (RF) peak voltage were set at $4000,150,65$, and $750 \mathrm{~V}$, respectively, for the scan source. The quadrupole was set to pass ions from $\mathrm{m} / z 100$ to 1000 . The MS scan rate was set to two spectra/s. Data were analyzed using Agilent MassHunter qualitative analysis software. Based on the molecular formula $\mathrm{C}_{13} \mathrm{H}_{28} \mathrm{~N}_{6} \mathrm{O}_{8}$ of $\mathrm{ZmA}$, the theoretical $m / z$ of $[\mathrm{M}+\mathrm{H}]^{+} 397.2046( \pm 20 \mathrm{ppm})$ was extracted to detect $\mathrm{ZmA}$ in each sample $[24,38]$.

\section{Additional file}

Additional file 1: Figure S1. Sequence analysis of the 24bp oriT core regions before (A) and after recombination (B). Figure S2. A test of the a-amylase activity of amyE mutant strain BMB0260. (A) and (B), Strain BMB171 and BMB0260 were grown on LB plates containing 1\% starch; One plate (B) was stained with iodine to detect the a-amylase activity, which was indicated by the Hydrolysis circle. 1, BMB171; 2-3, BMB0260. Figure S3. Cry5Ba and Cry2Aa expression in the unmarked cry gene integration mutants of BMB171. (A). Phase contrast microscopy graph of parasporal crystals in the unmarked cry gene integrate mutants. The arrows respectively indicates the diamond-shaped crystals encoded by cry $5 \mathrm{Ba}$ and the round-shaped crystals encoded by cry $2 \mathrm{Aa}$. (B). SDS-PAGE analysis of crystal proteins in the unmarked cry gene integration mutants of $\mathrm{BMB} 171$. The arrows indicate the bands produced by cry $5 \mathrm{Ba}$ and cry $2 \mathrm{Aa}$ respectively. 1, BMB171/cry5Ba-pHT304; 2-3, BMB0261; 4, YBT-1518; 5, BMB171/cry2Aa-pHT304; 6-7, BMB0262; 8, CT-43; 9, BMB171. M, Protein ladder.

\section{Authors' contributions}

PW, Y Zhu and MS designed the study, analyzed the data and wrote the manuscript. PW, Y Zhu, Y Zhang, CZ, JX and YD participated in the experiments, and $D P$ and LR critically reviewed the manuscript. All authors read and approved the final manuscript.

\section{Acknowledgements}

This work was supported by Grants from China 948 Program of Ministry of Agriculture (2016-X21 and 2011-G25), and the National Natural Science Foundation of China (31270137 and 31170047), and the National High Technology Research and Development Program (863) of China (2011AA10A203).

\section{Competing interests}

The authors declare that they have no competing interests.

Received: 16 February 2016 Accepted: 23 May 2016 Published online: 10 June 2016
References

1. Jensen GB, Hansen BM, Eilenberg J, Mahillon J. The hidden lifestyles of Bacillus cereus and relatives. Environ Microbiol. 2003;5:631-40.

2. Guinebretiere MH, Auger S, Galleron N, Contzen M, De Sarrau B, De Buyser ML, Lamberet G, Fagerlund A, Granum PE, Lereclus D, et al. Bacillus cytotoxicus sp. nov. is a novel thermotolerant species of the Bacillus cereus group occasionally associated with food poisoning. Int I Syst Evol Microbiol. 2013;63:31-40.

3. Jimenez G, Urdiain M, Cifuentes A, Lopez-Lopez A, Blanch AR, Tamames J, Kampfer P, Kolsto AB, Ramon D, Martinez JF, et al. Description of Bacillus toyonensis sp. nov., a novel species of the Bacillus cereus group, and pairwise genome comparisons of the species of the group by means of ANI calculations. Syst Appl Microbiol. 2013;36:383-91.

4. Xin B, Zheng J, Xu Z, Song X, Ruan L, Peng D, Sun M. The Bacillus cereus group is an excellent reservoir of novel lanthipeptides. Appl Environ Microbiol. 2015;81:1765-74.

5. Zhu L, Peng D, Wang Y, Ye W, Zheng J, Zhao C, Han D, Geng C, Ruan $\mathrm{L}$, He J, et al. Genomic and transcriptomic insights into the efficient entomopathogenicity of Bacillus thuringiensis. Sci Rep. 2015;5:14129.

6. Kopke K, Hoff B, Kuck U. Application of the Saccharomyces cerevisiae FLP/FRT recombination system in filamentous fungi for marker recycling and construction of knockout strains devoid of heterologous genes. Appl Environ Microbiol. 2010;76:4664-74.

7. Hoang TT, Karkhoff-Schweizer RR, Kutchma AJ, Schweizer HP. A broadhost-range Flp-FRT recombination system for site-specific excision of chromosomally-located DNA sequences: application for isolation of unmarked Pseudomonas aeruginosa mutants. Gene. 1998;212:77-86.

8. Song $\mathrm{H}$, Niederweis M. Functional expression of the Flp recombinase in Mycobacterium bovis BCG. Gene. 2007;399:112-9.

9. Fukiya S, Mizoguchi H, Mori H. An improved method for deleting large regions of Escherichia coli K-12 chromosome using a combination of Cre/loxP and lambda Red. FEMS Microbiol Lett. 2004;234:325-31.

10. Pomerantsev AP, Sitaraman R, Galloway CR, Kivovich V, Leppla SH. Genome engineering in Bacillus anthracis using Cre recombinase. Infect Immun. 2006;74:682-93.

11. Cascioferro A, Boldrin F, Serafini A, Provvedi R, Palu G, Manganelli R. Xer site-specific recombination, an efficient tool to introduce unmarked deletions into mycobacteria. Appl Environ Microbiol. 2010;76:5312-6.

12. Leroux M, Rezoug Z, Szatmari G. The Xer/dif site-specific recombination system of Campylobacter jejuni. Mol Genet Genomics. 2013;288:495-502.

13. Bloor AE, Cranenburgh RM. An efficient method of selectable marker gene excision by Xer recombination for gene replacement in bacterial chromosomes. Appl Environ Microbiol. 2006;72:2520-5.

14. Le Bourgeois P, Bugarel M, Campo N, Daveran-Mingot ML, Labonte J, Lanfranchi D, Lautier T, Pages C, Ritzenthaler P. The unconventional Xer recombination machinery of Streptococci/Lactococci. PLoS Genet. 2007;3:e117.

15. Garcillan-Barcia MP, Francia MV, de la Cruz F. The diversity of conjugative relaxases and its application in plasmid classification. FEMS Microbiol Rev. 2009;33:657-87.

16. Furuya N, Komano T. NikAB- or NikB-dependent intracellular recombination between tandemly repeated oriT sequences of plasmid R64 in plasmid or single-stranded phage vectors. J Bacteriol. 2003;185:3871-7.

17. Llosa M, Bolland S, Grandoso G, de la Cruz F. Conjugation-independent, site-specific recombination at the oriT of the IncW plasmid R388 mediated by TrwC. J Bacteriol. 1994;176:3210-7.

18. Meyer R. Site-specific recombination at oriT of plasmid R1162 in the absence of conjugative transfer. J Bacteriol. 1989;171:799-806.

19. Wang P, Zhang C, Zhu Y, Deng Y, Guo S, Peng D, Ruan L, Sun M. The resolution and regeneration of a cointegrate plasmid reveals a model for plasmid evolution mediated by conjugation and oriT site-specific recombination. Environ Microbiol. 2013;15:3305-18.

20. DominguezW, O'Sullivan DJ. Developing an efficient and reproducible conjugation-based gene transfer system for bifidobacteria. Microbiology. 2013;159:328-38.

21. Wang P, Yu Z, Li B, Cai X, Zeng Z, Chen X, Wang X. Development of an efficient conjugation-based genetic manipulation system for Pseudoalteromonas. Microb Cell Fact. 2015;14:11.

22. Kvitko BH, McMillan IA, Schweizer HP. An improved method for oriTdirected cloning and functionalization of large bacterial genomic regions. Appl Environ Microbiol. 2013;79:4869-78. 
23. Guo S, Liu M, Peng D, Ji S, Wang P, Yu Z, Sun M. New strategy for isolating novel nematicidal crystal protein genes from Bacillus thuringiensis strain YBT-1518. Appl Environ Microbiol. 2008;74:6997-7001.

24. Kevany BM, Rasko DA, Thomas MG. Characterization of the complete zwittermicin A biosynthesis gene cluster from Bacillus cereus. Appl Environ Microbiol. 2009;75:1144-55.

25. Peng D, Luo Y, Guo S, Zeng H, Ju S, Yu Z, Sun M. Elaboration of an electroporation protocol for large plasmids and wild-type strains of Bacillus thuringiensis. J Appl Microbiol. 2009;106:1849-58.

26. Turgeon N, Laflamme C, Ho J, Duchaine C. Elaboration of an electroporation protocol for Bacillus cereus ATCC 14579. J Microbiol Methods. 2006;67:543-8.

27. Widner WR, Whiteley HR. Location of the dipteran specificity region in a lepidopteran-dipteran crystal protein from Bacillus thuringiensis. J Bacteriol. 1990;172:2826-32.

28. Draper O, Cesar CE, Machon C, de la Cruz F, Llosa M. Site-specific recombinase and integrase activities of a conjugative relaxase in recipient cells. Proc Natl Acad Sci USA. 2005;102:16385-90.

29. Sanchis V, Agaisse H, Chaufaux J, Lereclus D. A recombinase-mediated system for elimination of antibiotic resistance gene markers from genetically engineered Bacillus thuringiensis strains. Appl Environ Microbiol. 1997;63:779-84

30. Francia MV, Clewell DB. Amplification of the tetracycline resistance determinant of pAMalpha1 in Enterococcus faecalis requires a site-specific recombination event involving relaxase. J Bacteriol. 2002;184:5187-93.

31. Gennaro ML, Kornblum J, Novick RP. A site-specific recombination function in Staphylococcus aureus plasmids. J Bacteriol. 1987;169:2601-10.

32. Sambrook J, Russell DW. Molecular cloning: a laboratory manual. 3rd ed. Cold Spring Harbor: Cold Spring Harbor Laboratory Press; 2001.

33. Dartois $V$, Djavakhishvili $T$, Hoch JA. Identification of a membrane protein involved in activation of the KinB pathway to sporulation in Bacillus subtilis. J Bacteriol. 1996;178:1178-86.
34. Tounsi S, Jaoua S. Characterization of a novel cry2Aa-type gene from Bacillus thuringiensis subsp. kurstaki. Biotechnol Lett. 2003;25:1219-23.

35. Zhu Y, Ji F, Shang H, Zhu Q, Wang P, Xu C, Deng Y, Peng D, Ruan L, Sun $M$. Gene clusters located on two large plasmids determine spore crystal association (SCA) in Bacillus thuringiensis subsp. finitimus strain YBT-020. PLOS ONE. 2011;6:e27164.

36. Wilcks A, Jayaswal N, Lereclus D, Andrup L. Characterization of plasmid pAW63, a second self-transmissible plasmid in Bacillus thuringiensis subsp. kurstaki HD73. Microbiology. 1998;144(Pt 5):1263-70.

37. Shao Z, Liu Z, Yu Z. Effects of the 20-kilodalton helper protein on Cry1AC production and spore formation in Bacillus thuringiensis. Appl Environ Microbiol. 2001;67:5362-9.

38. Luo Y, Ruan LF, Zhao CM, Wang CX, Peng DH, Sun M. Validation of the intact zwittermicin A biosynthetic gene cluster and discovery of a complementary resistance mechanism in Bacillus thuringiensis. Antimicrob Agents Chemother. 2011;55:4161-9.

39. He J, Shao X, Zheng H, Li M, Wang J, Zhang Q, Li L, Liu Z, Sun M, Wang S, Yu Z. Complete genome sequence of Bacillus thuringiensis mutant strain BMB171. J Bacteriol. 2010;192:4074-5.

40. He J, Wang J, Yin W, Shao X, Zheng H, Li M, Zhao Y, Sun M, Wang S, Yu Z. Complete genome sequence of Bacillus thuringiensis subsp. chinensis strain CT-43. J Bacteriol. 2011;193:3407-8.

41. Liu XY, Ruan LF, Hu ZF, Peng DH, Cao SY, Yu ZN, Liu Y, Zheng JS, Sun M. Genome-wide screening reveals the genetic determinants of an antibiotic insecticide in Bacillus thuringiensis. J Biol Chem. 2010;285:39191-200.

42. Wang P, Zhang C, Guo M, Guo S, Zhu Y, Zheng J, Zhu L, Ruan L, Peng D, Sun M. Complete genome sequence of Bacillus thuringiensis YBT-1518, a typical strain with high toxicity to nematodes. J Biotechnol. 2014;171:1-2.

43. Arantes $\mathrm{O}$, Lereclus D. Construction of cloning vectors for Bacillus thuringiensis. Gene. 1991;108:115-9.

\section{Submit your next manuscript to BioMed Central and we will help you at every step:}

- We accept pre-submission inquiries

- Our selector tool helps you to find the most relevant journal

- We provide round the clock customer support

- Convenient online submission

- Thorough peer review

- Inclusion in PubMed and all major indexing services

- Maximum visibility for your research

Submit your manuscript at www.biomedcentral.com/submit
O Biomed Central 